Tissue Engineering

\title{
Wie Licht beim Aufbau von Geweben helfen kann
}

MARC MÜLLER ${ }^{1}$, SERAPHINE V. WEGNER ${ }^{2}$

${ }^{1}$ MAX-PLANCK-INSTITUT FÜR POLYMERFORSCHUNG, MAINZ

2 INSTITUT FÜR PHYSIOLOGISCHE CHEMIE UND PATHOBIOCHEMIE, UNIVERSITÄT MÜNSTER

\section{Building tissues from cells as their basic building block is a promising approach in tissue engineering and allows assembling cells into micro- tissues with high precision, which is not possible with material-based approaches. The challenge lies in controlling when and where cells bind to each other. Using visible light as a trigger for cell-cell interac- tions provides the required spatiotemporal control without interfering with other cellular processes. This provides a new way to assemble multicellular structures with numerous potential applications in cell biology and regenerative medicine.}

DOI: $10.1007 / \mathrm{s} 12268-020-1412-4$

(C) Die Autoren 2020

Das Züchten von Geweben und Organen im Labor ist ein vielversprechender Ansatz, um der zurzeit herrschenden Organknappheit entgegenzuwirken. Im Bereich des Gewebeaufbaus, auch Tissue Engineering genannt, werden Ersatzgewebe hergestellt, die das beschädigte Gewebe reparieren, wiederaufbauen oder verbessern können. Ein neuer Ansatz zur Gewebezüchtung ist das bottom-up Tissue Engineering, bei dem Zellen als Grundbausteine des Gewebes verwendet und wie Puzzleteile zu einem neuen Gewebe zusammengebaut werden (Abb. 1A). Dieser Ansatz setzt auf das natürliche Potenzial von Zellen, sich selbst in größeren und komplexeren Strukturen anordnen zu können [1]. Diese Eigenschaft ist besonders vorteilhaft, um feine Mikrostrukturen aufzubauen, die mit traditionellem Tissue Engineering nicht hergestellt werden können.

Ein Gewebe aus einzelnen Zellen aufzubauen, klingt allerdings einfacher, als es ist, da es nicht ausreicht, die verschiedenen Zelltypen, die ein Gewebe bildet, miteinander zu vermischen. Sie müssen auch räumlich richtig angeordnet sein und miteinander kommunizieren. Nur wenn all diese Faktoren berücksichtigt werden, kann ein funktionsfähiges Gewebe entstehen.
Wie bei einem 3D-Puzzle ist die Anordnung der unterschiedlichen Zellen wichtig und abhängig von den einzelnen Zell-ZellInteraktionen. Einmal richtig organisiert, können Zellen ihre eigene extrazelluläre Matrix bilden, wachsen, sich differenzieren und somit feinstrukturierte Mikrogewebe erzeugen, die für den Aufbau funktionaler Gewebe unerlässlich sind. scheidet sich von den in der Gewebezüchtung gängigen Ansätzen. Bei den bisherigen Verfahren besiedeln Zellen ein künstliches Gerüst, auf dem sie sich vermehren, differenzieren und so das gewünschte Gewebe bilden. Das künstliche Material gibt dabei die Form für das Gewebe vor und bietet mechanische Stabilität. Die Zellen können sowohl mit Adhäsionsmolekülen als auch mit wasserlöslichen Wachstumsfaktoren stimuliert werden. Allerdings ist es eine Herausforderung, aus den Gerüstmaterialien kleine, sehr präzise Strukturen im Mikrometerbereich herzustellen, auf denen dann mehrere Zelltypen kontrolliert angesiedelt werden können. Das bottom-up Tissue Engineering überwindet genau diese Probleme, indem es direkt aus Zellen feinstrukturierte Mikrogewebe aufbaut.
Das bottom-up Tissue Engineering unter-
Mit der Kombination der beiden Vorgehensweisen können richtig angeordnete Mikrogewebe in Gerüste mit großen Strukturen und hoher mechanischer Stabilität eingebettet werden [2]. Somit ergänzen sich die beiden Ansätze, um komplexere Gewebe herzustellen.

\section{Kontrollierte Zell-Zell-Kontakte}

Um Gewebe nach dem bottom-up-Prinzip aufbauen zu können, ist es hilfreich, die Prozesse der Entstehung von Geweben und Organismen in der Natur zu verstehen. Bereits in den 1960er-Jahren setzte sich Malcom Steinberg mit der Frage auseinander, wie sich Strukturen in multizellulären Organismen bilden [3]. Es war bereits bekannt, dass während der Embryonalentwicklung Zellen oder Gruppen von Zellen von ihren Ursprungsorten zu ihren korrekten anatomischen Positionen wandern. In frühen Experimenten wurden Gewebe in einzelne Zellen dissoziiert und später verschiedene Zelltypen neu zusammengemischt. Dabei wurde festgestellt, dass Zellen vom gleichen Typ an ihren richtigen Ort wanderten und dort erneut aggregierten, um dieselbe Position wie auch im Ausgangsgewebe einzunehmen. Diese Experimente zeigten, dass die Organisation eines Gewebes durch dauerhaft vorhandene Kräfte vermittelt wird und nicht aus der chronologischen Abfolge der vorangegangenen Entwicklungsereignisse resultiert. Steinberg fand heraus, dass diese Kräfte durch ZellZell-Adhäsionen vermittelt werden und diese die Struktur des Gewebes und somit die Organisation der unterschiedlichen Zelltypen vorgeben [4]. Damit diese Selbstorganisation möglich ist, müssen die Interaktionen zwischen den Zellen dynamisch sein. Nur so ist die Bewegung der Zellen relativ zueinander und somit das Sortieren trotz starker ZellZell-Kontakte möglich. Damit stellte sich heraus, dass die räumliche und zeitliche Regulierung der interzellulären Adhäsion den Aufbau des Gewebes bestimmt und zur Positionierung am richtigen Ort führt.

Bei der Entwicklung künstlicher Gewebe müssen die Interaktionen zwischen den Zellen 


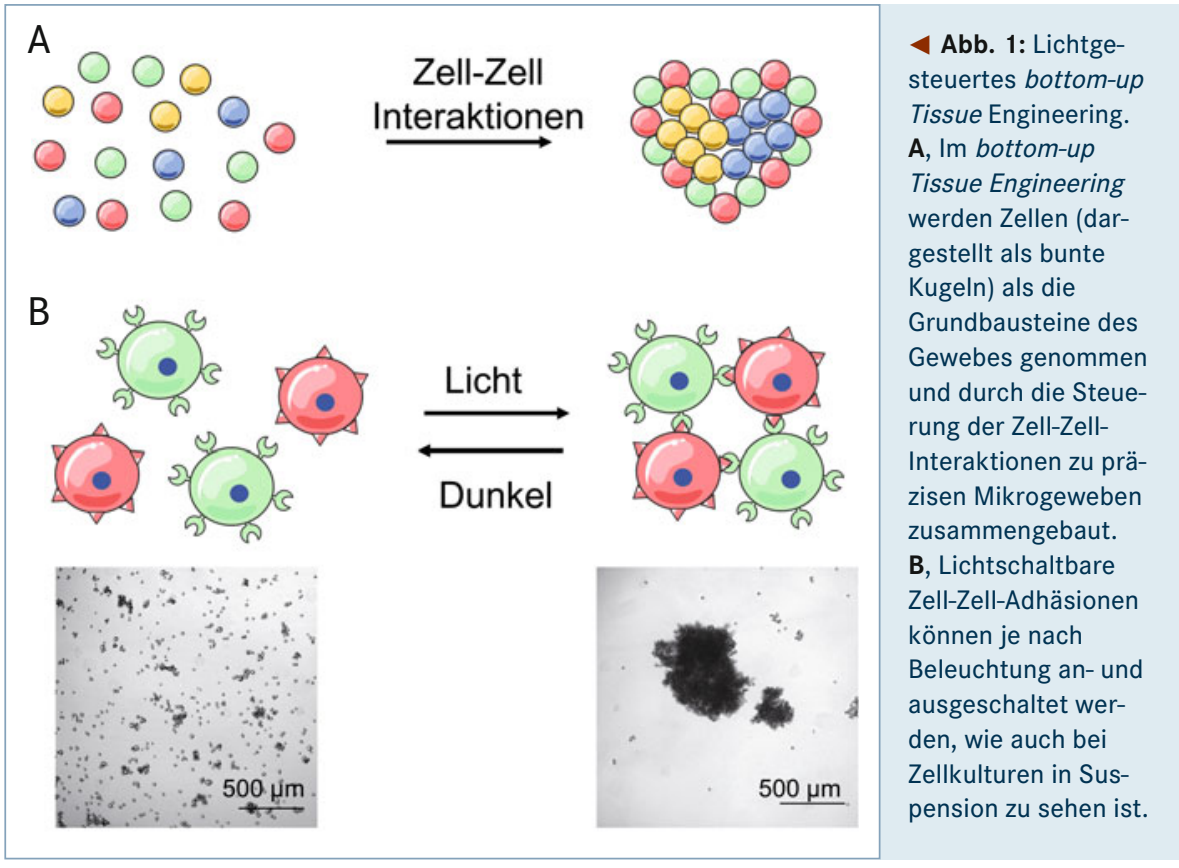

kontrolliert werden. Das kann über die Expression von Adhäsionsmolekülen (z. B. Cadherine) auf der Zelloberfläche geregelt werden, allerdings verändert dies auch die Expression von weiteren Genen und somit die ganze Zelle. Eine bioorthogonale Möglichkeit, Zell-Zell-Interaktionen zu kontrollieren, sind chemische Moleküle, die auf der Zelloberfläche angebracht werden und mit Molekülen auf benachbarten Zellen reagieren können. Zu diesem Zweck wurden besonders milde Reaktionen genutzt, die andere Prozesse in der Zelle nicht beeinflussen und im Wasser ablaufen, wie z. B. Klick-Chemie [5] und komplementäre DNA-Stränge [6]. Die gezielte Modifikation unterschiedlicher Zellen erlaubt es somit, Zellen permanent über starke Bindungen aneinanderzubinden.

Aber auch diese Strategie hat ihre Grenzen: Zum einen werden die Zellen durch die Modifikation zusammengeklebt, wobei die Dynamik der Zell-Zell-Adhäsionen verloren geht. Zum anderen können die Zell-Zell-Kontakte nicht zu einem bestimmten Zeitpunkt bzw. an einer bestimmten Stelle aktiviert werden, sodass eine räumliche oder zeitliche Regulierung nicht möglich ist. Ein weiteres Problem ist, dass die chemischen Modifikationen auf der Zelloberfläche mit der Zeit abgebaut und bei jeder Zellteilung weiter verdünnt werden.

\section{Steuerung mit Licht}

In unserem Labor werden seit einigen Jahren Zell-Zell-Kontakte mit Licht gesteuert, um sie in Raum und Zeit kontrollierbar zu machen.
Das heißt, dass Zellen so verändert werden, dass sie unter dem Einfluss von Licht aneinanderbinden und multizelluläre Strukturen bilden (Abb. 1B).

Dieses Verfahren bringt deutliche Vorteile. Erstens ermöglicht die Regulierung der Zellkontakte durch Licht, die Wechselwirkungen zwischen den Zellen zu einem beliebigen Zeitpunkt zu aktivieren, indem einfach nur das Licht angeschaltet wird. Umgekehrt ist es auch möglich, Adhäsionen wieder zu lösen, indem das Licht ausgeschaltet wird.

Zweitens können Zellen durch eine lokale Beleuchtung in einem definierten Bereich aktiviert werden, ohne dass Zellen in anderen Bereichen interagieren. Die beleuchtete Fläche kann dabei wenige Mikrometer betragen, also kleiner als die einzelne Zelle sein. Es ist aber auch möglich, komplexe Lichtmuster über Photomasken auf große Bereiche mit vielen Zellen zu projizieren. Licht ist ein geeigneter Reiz, um Zellen zu steuern und ohne direkten Kontakt in das Zellkultursystem einzubringen. Weitere Vorteile der Regulierung der Zelladhäsionen mit Licht über die hohe zeitliche und räumliche Kontrolle hinaus sind, dass die Stärke der Adhäsionen und deren Dynamik über die Beleuchtungsparameter, wie Lichtintensität und Beleuchtungsfrequenz, eingestellt werden kann.

Um Zellinteraktionen mit Licht zu steuern, verwenden wir lichtschaltbare Proteine als Adhäsionsmoleküle (Abb. 1B, [7]). Dabei werden Zellen genetisch so modifiziert, dass sie diese Proteine selbst produzieren und auf ihrer Zelloberfläche präsentieren. Wenn zwei Zelltypen, die komplementäre lichtschaltbare Proteine auf ihrer Oberfläche exprimieren, im Dunkeln gemischt werden, bilden sie zunächst keine Kontakte, da die Proteine ohne Licht nicht aneinanderbinden. Wenn man dann dieses Zellgemisch beleuchtet, werden die Wechselwirkungen zwischen den Proteinen aktiviert und es bilden sich Zell-Zell-Interaktionen. Diese photoschaltbaren Zell-Zell-Adhäsionen führen dazu, dass unter Beleuchtung Zellen auf Oberflächen in Gruppen und nicht einzeln wachsen. Auch in Zellsuspensionen bilden sich unter Licht große Zellaggregate. Diese lichtschaltbaren Proteine, die auf der Zelloberfläche verankert sind, fungieren somit als künstliche Adhäsionsmoleküle und sind, anders als die zelleigenen Adhäsionsmoleküle, nicht mit intrazellulären Signalwegen verbunden.

Eine Vielzahl an lichtschaltbaren Proteinen gestattet es, unterschiedliche Zell-ZellKontakte zu vermitteln und diese unabhängig voneinander mit Licht unterschiedlicher Wellenlänge zu steuern. Diese lichtsensitiven Proteine stammen ursprünglich aus Pflanzen und photosynthetischen Organismen und wurden über das letzte Jahrzehnt im Bereich der Optogenetik für die Kontrolle von Zellen mit Licht entwickelt.

Manche dieser Proteine gehen unter Licht eine Bindung mit sich selbst ein - sie homodimerisieren - und sind somit geeignet, Zellen des gleichen Typs, die diese Proteine auf der Oberfläche exprimieren, zusammenzubringen (Abb. 2A, [8]). Die andere Variante sind Proteinpaare, die bei Beleuchtung aneinanderbinden, also heterodimerisieren. Zwei unterschiedliche Zelltypen, die je einen der Bindungspartner auf der Oberfläche präsentieren, können durch Lichteinwirkung miteinander verbunden werden [7, 9]. Mit diesen unterschiedlichen selbst- und fremdspezifischen Zell-Zell-Interaktionen können wir gezielt Zellen vom gleichen oder unterschiedlichen Typus in einer multizellulären Struktur zusammenbauen.

Um in einem Gemisch unterschiedliche Zell-Zell-Adhäsionen unabhängig voneinander zu steuern, bedarf es zweier Stimuli, die sich gegenseitig nicht beeinflussen. Hier bieten die lichtschaltbaren Adhäsionen die Möglichkeit, Proteine zu verwenden, die auf unterschiedliche Wellenlängen reagieren [8]. Bei unseren Versuchen arbeiten wir mit zwei unterschiedlichen Zelltypen: Der eine Zelltyp 


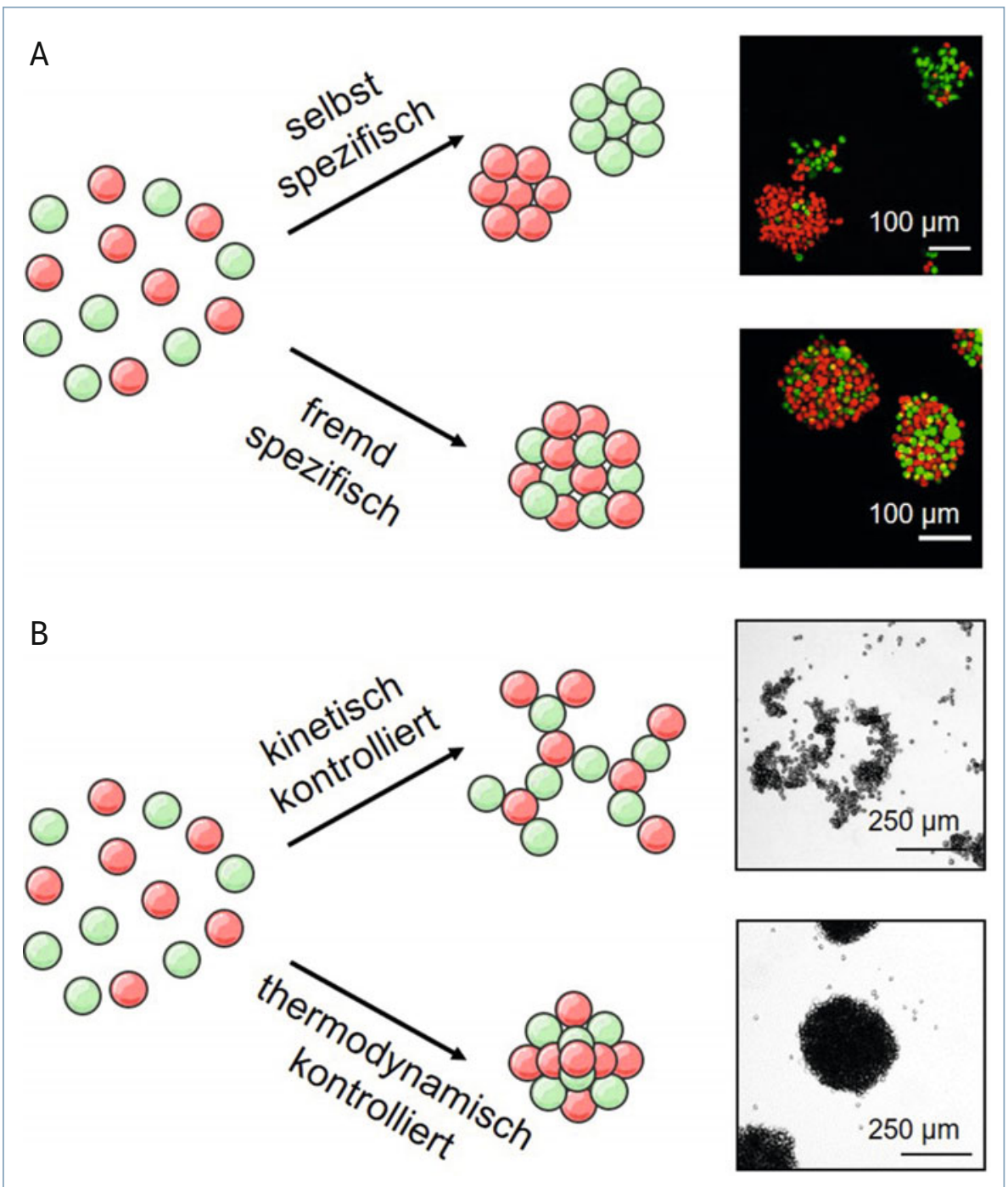

$\Delta$ Abb. 2: Anordnung der Zellen in multizellulären Strukturen. A, Je nachdem, ob die Proteine auf den Zellen homo- oder heterodimerisieren, bilden sich multizelluläre Strukturen aus gleichen oder unterschiedlichen Zelltypen. Dies ist anhand der grün und rot fluoreszenzmarkierten Zellen zu sehen. B, Die Dynamik der Zell-Zell-Interaktionen bestimmt, ob sich kinetisch kontrollierte, verzweigte Strukturen oder thermodynamisch kontrollierte, kompakte Strukturen bilden.

reagiert auf blaues $(480 \mathrm{~nm})$ und der andere auf rotes Licht (660 nm). So konnten wir gezielt die Zell-Zell-Interaktionen des einen Zelltyps aktivieren, ohne den anderen zu beeinflussen. Die hohe Spezifizität einzelner Protein-Protein-Wechselwirkungen erlaubt es, auch diese Zelltypen zu sortieren; das heißt bei Beleuchtung mit blauem und rotem Licht, bei der beide Adhäsionen aktiviert werden, aggregieren die beiden Zellarten nicht in einer Struktur, sondern jeder Zelltyp sondert sich ab und bildet ein separates Aggregat. Dieses Selbstsortierungsverhalten der Zellen wird auch in der Embryonalentwicklung beobachtet und kann über die blauund rotlichtgesteuerten Zell-Zell-Adhäsionen kontrolliert werden.
Ein weiterer Aspekt, der bei der Herstellung von Gewebestrukturen eine wichtige Rolle spielt, ist die Dynamik der Zell-ZellAdhäsionen (Abb. 2B, [9]). Wenn Zellen sehr stark miteinander interagieren und daher nach den ersten Kontakten nicht die Möglichkeit haben, sich neu anzuordnen, bilden sich verzweigte, multizelluläre Strukturen. Diese werden als kinetisch kontrollierte Strukturen bezeichnet. Theoretisch haben diese Zellen die Möglichkeit, noch weitere Kontakte zu anderen Zellen zu bilden. Durch die starke Interaktion ist dies jedoch nicht mehr möglich, da sie kinetisch gefangen sind. Sind die Zell-Zell-Kontakte hingegen dynamisch, können die Zellen sich anordnen und somit ihre Kontakte maximieren. Dabei entstehen kom- pakte, runde Strukturen, die thermodynamisch kontrolliert sind. Ob kinetisch oder thermodynamisch kontrollierte Strukturen entstehen, ist abhängig von den Adhäsionsmolekülen und in unserem Fall von den photoschaltbaren Proteinen. Diese unterschiedlichen Dynamiken können für eine unterschiedliche Anordnung der Zellen in einer multizellulären Struktur genutzt werden. Zusätzlich kann durch ein gezieltes An- und Ausschalten des Lichts die Dynamik erhöht werden. Eine pulsierende Lichtaktivierung erlaubt den Zellen, sich jedes Mal neu anzuordnen und bei dem nächsten Lichtpuls zu binden. So können kinetisch kontrollierte Strukturen in Richtung thermodynamisch kontrollierter Strukturen verschoben werden.

\section{Zusammenfassung}

Der Aufbau von künstlichem Gewebe aus einzelnen Zellen ist ein vielversprechender, jedoch auch äußerst herausfordernder Forschungsansatz, um komplexe Strukturen herzustellen. Dieser Ansatz, der Zellen als Grundbausteine des Gewebes verwendet, ist besonders vorteilhaft, um feine Mikrostrukturen aufzubauen, die mit traditionellem Tissue Engineering nicht hergestellt werden können. Die Herausforderung liegt dabei in der Steuerung der Zell-Zell-Interaktionen, die bestimmen, wie sich Zellen zueinander organisieren, wie sie zusammenarbeiten und in der Klärung der Frage, ob eine solche vielzellige Gewebearchitektur funktionsfähig ist.

In unseren Arbeiten werden lichtschaltbare Proteine als Adhäsionsmoleküle auf der Zelloberfläche angebracht, um die Zellinteraktionen mit Licht steuern zu können. Hierbei ermöglichen die photoschaltbaren Adhäsionen eine einzigartige, nicht invasive und bioorthogonale Fernsteuerung der Zellen, mit hoher räumlicher und zeitlicher Genauigkeit. Zeitlich variable lokale Lichtimpulse auf unterschiedliche Zellen führten bereits erfolgreich zum Aufbau von multizellulärem Mikrogewebe mit einmaliger Präzision. Diese Ansätze bieten neue Möglichkeiten für den Gewebeaufbau in der regenerativen Medizin.

\section{Literatur}

[1] Wan ACA (2018) Recapitulating cell-cell interactions for organoid construction - are biomaterials dispensable? Trends Biotechnol 36:711-721

[2] Ovsianikov A, Khademhosseini A, Mironov V (2018) The synergy of scaffold-based and scaffold-free tissue engineering strategies. Trends Biotechnol 36:348-357 
[3] Steinberg MS (1963) Reconstruction of tissues by dissociated cells. Science 141:401-408

[4] Duguay D (2003) Cadherin-mediated cell adhesion and tissue segregation: qualitative and quantitative determinants. Dev Biol 253:309-323

[5] Dutta D (2011) Synthetic chemoselective rewiring of cell surfaces: generation of three-dimensional tissue structures. J Am Chem Soc 133:8704-8713

[6] Zev J, Gartner ZJ, Bertozzi CR (2009) Programmed assembly of 3-dimensional microtissues with defined cellular connectivity. Proc Natl Acad Sci USA 106:4606-4610

[7] Yüz SG (2019) Blue light switchable cell-cell interactions provide reversible and spatiotemporal control towards bottom-up tissue engineering. Adv Biosys 3:1800310 [8] Sentürk OI (2019) Independent blue and red light triggered narcissistic self-sorting self-assembly of colloidal particles. Small 15:e1901801

[9] Mueller M (2020) The importance of cell-cell interaction dynamics in bottom-up tissue engineering: concepts of collodal self-assembly in the fabrication of multicellular architectures. Nano Lett 20:2257-2263
Funding: Open Access funding provided by Projekt DEAL.

Open Access: Dieser Artikel wird unter der Creative Commons Namensnennung 4.0 International Lizenz veroffentlicht, welche die Nutzung, Vervielfältigung,

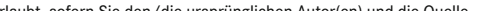
ordnungsgemäß nennen, einen Link zur Creative Commons Lizenz beifügen und

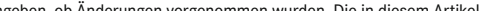
nthaltenen Bilder und sonstiges Drittmaterial unterliegen ebenfalls der genannten Creative Commons Lizenz, sofern sich aus der Abbildungslegende nichts anderes ergibt. Sofern das betreffende Material nicht unter der genannten Creative Commons Lizenz steht und die betreffende Handlung nich ach gesetzlichen Vorschriften erlaubt ist, ist für die oben aufgeführten Weiterverwendungen des Materials die Einwilligung des jeweiligen Reohteinhabers einzuholen. Weilere Details zur Lizenz enthehmen Sie bitte der

Korrespondenzadresse:

Prof. Dr. Seraphine Wegner

Institut für Physiologische Chemie und

Pathobiochemie

Universität Münster

Waldeyerstraße 15

D-48149 Münster

wegnerse@exchange.wwu.de

Marc Müller
2010-2013 Bachelorstudium Biologie am Karlsruher Institut für Technologie (KIT).
$2013-2016$ Masterstudium Biologie an der Universität Freiburg. 2016-2019 Promo-
tion am Max-Planck-Institut für Polymerforschung, Mainz, in der synthetischen Bio-
logie.

\section{Hier steht eine Anzeige.}

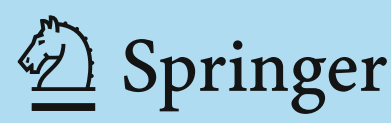

